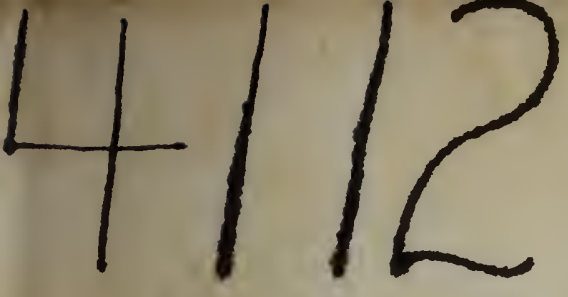

ACADÉMIE DE PARIS.

PACOLTE DES SGIENGES.

\title{
REMARQUES
}

SUR LES OISEAUX FOSSILES.

\section{THĖSE DE GÉOLOGIE}

BOUTENUE

DEVANT LA FAGULTÉ DES SCIENGES DE PARIS,

Le 5 Août, 1844.

POUR ÎTRE ADMIS AU GRADE DE DOCTEUR ÈS-SCIENCES

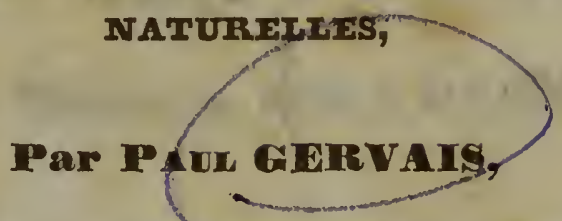

Licencié ès-sciences, Employé àtr-Muséum d'histoire naturelle de Paris, Membre de la Société Philomatique.

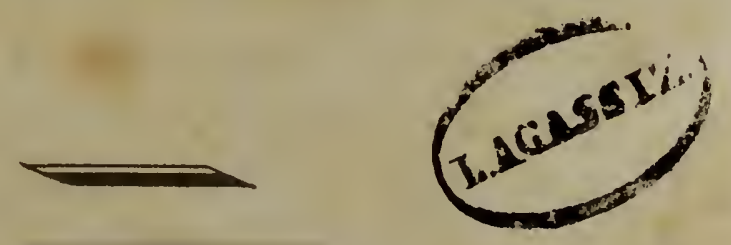

\section{PARIS,}

IMPRIMERIE DE POUSSIELGUE,

$$
\text { RUE DU CROISSANT, } 12 .
$$

i) 1844 


\section{Mato on I}

incule of $x$

. 
SUR

\section{LES OISEAUX FOSSILES.}

Les animaux mammifères ont laissé dans les couches tertiaires du globe des os nombreux et des dents dont on pe'st, dans la plupart des cas, reconnaître la nature avec assez de certitude pour indiquer si l'espèce à laquelle ils ont appartenu différait ou non de celles de l'époque actuelle. Les plaques osseuses qui recouvraient le corps de plusieurs de ces mammifères, les écailles plus ou moin: résistantes des poissons, les dents et certains os des reptiles, des amphibiens ou des poissons, ont conduit à des résultats analogues. Il n'en est pas toujours ainsi pour les Ornitholithes, c'est à dire pour les restes fossiles par lesquels nous cherchons à déterminer quels oiseaux existaient aux diverses époques dela vie du globe. Les ossements des viseaux permettent des résultats beaucoup moins positifs dans beaucoup de cas, et G. Cuvier en exprime la raison principale lorsqu'il dit :

a Les oiseaux se ress mblent entre eux beaucoup plus que les quadrupèdes; $l i s$ limites extrêmes de la classe sont plus rapprochées, et le numbre des espèces renfermées entre ces limites beaucoup plus considérable. Les diflé- 
rences entre deux espèces seront donc quelquefois inappréciables dans le squelette. Les genres mêmes n'ont pas toujours des caractères ostéologiques suffisants. n (1)

En effet, la détermination des ossements fossiles d'oiseaux est le plus ordinairement impossible quand on veut s'assurer de l'espèce elle-même à laquelle ils appartiennent, car ici les parties sur lesquelles porte habituellement la différence des espèces d'un mêrne genre ne sont pas susceptibles de conservation. Le bec, l'écaillure des paites, le plumage, tout a disparu par la fossilisation; les os, fort souvent altérés, ne conduisent pas tous à des diagnoses génériques, et nulle pièce ne remplace les dents, les carapaces ou les écailles des autres classes d'animaux vertébrés; toutes parties si aisément reconnaissables qu'on a presque établi d'une manière définitive les lois de leurs variations en rapport avec la condition classique et biologique des nombreuses espèces dont elles ont fait partie.

"Je le répète (dit Cuvier, en terminant son mémoire sur ies ornitholithes de Montmartre, dont il avait cependant démontré la multiplicité d'espèces, mais à propos des affinités qu'il assigne à quelques-unes d'entre elles), et les naturalistes le sentiront assez sans que je le dise, ce ne sont là que des conjectures qui sont bien éloignées d'être aussi certaines que mes propositions relatives aux os de quadrupèdes. C'est bien assez d'avoir montré l'existence de la classe des oiseaux parmì les fossiles, et d'avoir prouvé par là qu'à cette époque reculée, où les espèces étaient si différentes de celles que nous voyons maintenant, les lois générales de coexistence, de structure, et enfin tout ce qui s'élève au dessus des simples rapports spécifiques, tout ce

(1) Oiscaux fossiles, MI, 524. 
qui tient à la nature même des organes et à leurs fonctions essentielles étaient les mêmes que de nos jours."

On connaissait, avant ce travail de Cuvier, quelques os fossiles d'oiseaux bien constatés, et depuis lors on a beaucoup ajouté d'indications à celles qu'il a lui-même données, et qui sont relatives aux fossiles des gypses parisiens. C'est à la discussion de ces nombreux documents, quelles que soient les espècés d'oiseaux dont ils proviennent, et les points du globe où ils aient été trouvés, que le présent mémoire est consacré.

J'appellerai Ornínolithes toutes les traces soit empreintes, plumes, œufs ou ossements, trouvées dans les couches diverses du globe, mais provenant d'une fossilisation naturelle et susceptibles de faire reconnaître l'existence d'oiseaux à la surface du globe aux époques antérieures à la nôtre, et j'en parlerai en suivant l'ordre géologique de leur gisement. Je dois aussi prévenir de la signification des deux mots Ornitichnites et Osteornis, par lesquels je désignerai deux sortes des témoins que la classe des oiseaux a laissés de son ancienne existence.

Osteornis. - C'est à dire os d'oiseau. Je nommerai ainsi les débris osseux ou les os entiers d'oiseaux trouvés à l'état fossile (1), et j'en proposerai l'emploi aux naturalistes, pour désigner tous les ornitholithes osseux dont la détermination spécifique paraîtra impossible. Je sais que beaucoup de paléontologistes, et même plusieurs anatomistes, dénomment souvent comme espèces distinctes des fossiles dont la caractéristique est cependant impossible, et qui ne consistent qu'en ossements au moyen desquels on ne saurait indiquer plus que le genre, même en laissant à celui.ci

(1) Lithosteornis serait préférable, mais c'est un mot trop long. 
toute l'extension que lui accordait linné. L'âge démontré ou supposé des terrains d'où proviennent ces fossiles est souvent la seule indication qui puisse faire supposer que les espèces ainsi nommées diffèrent ou ne diffèrent pas de celles des couches adjacentes, ou de l'époque actuelle; aussi dans ce cas la théorie seule est-elle consultée, et les uns établissent-ils l'identité, tandis que d'autres la nient formellement. Le zoologiste invoque l'auturité du géologue, qui allègue à son tour celle du zoologiste, et somme toute la démonstration de l'un et de l'autre n'est autre chose qu'une pétition de principes. La nomenclature linnéenne a trop de précision pour que nous puissions l'employer constamment en paléontologie. Quand on a des doutes sur le genre d'un oiseau, comment assurer son espèce, et alors pourquoi lui donner un nom spécifique; quand on n'arrive qu'à la détermination du genre. pourquoi faire dire ì la science plus qu'elle n'est en droit de le faire en suivant pour la dénomination d'êtres aussi difficiles à reconnaitre les mêmes règles que pour les animaux vivants ou que l'on possède entiers, tandis qu'on n'a de ceux qui ont cessé d'exister aux époques anté-historiques que des débris le plus souvent incomplets. Tout os fossile d'oiseau sera donc un Osteornis. On rappellera par ce nom seul un fait important pour la paléontologie, l'existence d'oiseaux fossiles à l'époque où tel terrain s'est formé; un qualificatif de ce nom collectif indiquera si l'ornitholithe est douleux, ou si la détermination qu'on en a faite indique, au con/raire, l'ordre, la fanille ou le genre auxquels son espèce appartenait; et, comme les oiseaux fossiles d'espèce indéterminée, quels que soient leur genre, leur famille ou leur ordre, recevront le mên e nom collectif, on pourra, si l'on use de ce moyen avec réserve, enregistrer les gisements d'oiscaux avec autant de précision que précédemment, et sans néanmoins encombrer les catalugues pa- 
léontologıques d'espèces purement nominales, tant qu'elles n'ont pas été démontrées et qu'on ne leur a pas assigné une place réelle dans la méthode. Les détails que nous donnerons bientôt montreront combien est encore restreint le nombre des ornitholithes que l'on connaît suffisamment pour en désigner l'espèce.

On pourrait appliquer de semblables noms aux ornitholithes qui consistent en œufs, ainsi qu'on en trouve en Auvergne, ou en plumes, comme plusieurs localités en ont fourni; mais on ne connaît point encore un nombre suffisant des uns et des autres pour qu'il soit nécessaire de leur imposer une dénomination spéciale, et ces dénominations seront très faciles à faire quand on en reconnaîtra le besoin.

Ornithichnites. - M. Hitchcock appelle ainsi un genre d'oiseaux qui aurait laissé dans le nouveau grès ronge du Connecticut des empreintes de pieds indiquant plusieurs espèces. (O. giganteus, tuberosus, ingens, etc.) Il n'a pas un seul ns de ces oiseaux; les différences de la grandeur des traces lui font supposer dans la taille des animaux qui les ont laissées des différences plus considérables que celles qu'on remarque entre l'Autruche d'Afrique et la Bécasse, et cependant M. Hitchcock fait des unes et des autres un même genre, et dans ce genre il établit sept espèces. Si nous étions bien convaincu que toutes ces traces proviennent d'oiseaux, nous les rapporterions à une catégorie spéciale d'ornithulithes, pour laquelle on pourrait conserver le nom qu'a inventé M. Hitchcock, bien qüil ne soit pas très euphonique; nous admettrions que ces traces sont de plusieurs sortes; qu'elles indiquent certainement plusieurs espèces. mais sans donner au nom désignatif de ces espèces la valeur des noms spécifiques de la nomenclature binaire, et toutes les traces ou pas fossiles d'oiseaux qui pourront être signalées, à quelque terrain qu'elles appartiennent, seraient également des ornithichnites. 


\section{CHAPITRE PREMIER.}

ORNITHOLITHES DES FORMATIONS SECONDAIRES.

\section{$\$ I$.}

Dans te grès-rouge.

Les traces les plus anciennes que l'on ait rapportées à des oiseaux sont ces Ornithichnites de la vallée de Connecticut, décrits par M Hitchcock (1) et dont nous venons de parler.

Ces empreintes se succèdent régulièrement, et résultent, suivant l'auteur, de la trace d'animaux dans l'acte de marcher ou de courir; le pied droit et le pied gauche se montrenı toujours à leur place respective. M. Hitchcock en reconnaît de sept sortes différentes, et qui toutes lui paraissent indiquer des Echassiers. La plupart ne montrent que trois doigts.

L'O. giganteus, Hitch., est l'empreinte la plus remarquable, et serait d'un oiseau deux fois grand comme l'Autruche. Les autres sont nommés $O$. tuberosus, ingens, diversus (partagé en deux variétés: $O$. clarus et platydactylus) tetradactylus, palmatus et minimus.

On a déjà émis bien des doutes sur les conclusions du

(1) American journal of sciences and arts, T. xxix, 1856. Ses figures et son lexle, en totalité ou en abrégé, sont reproduits par: Buckland, Gíologic el miner., II, pl. 26 a el b.-Ann. sc. nat. $2^{\mathrm{e}}$ Séric, T. V, p. 154, pl. 6.-Bronı et Léonard, Neues iarbuch, 1856, pl. 5. - Pictet, l'alcontologic, T. I, 1844 ; etc. 
travail de M. Hitchcock, et plusieurs de ces doutes conservent toute leur valeur. M. Pictet refuse d'accepter l'O. minimus comme véritable ornithichnite, et l'on peut aussi demander à propos de l'O. ingens, s'il est raiionnel d'admettre, avec M. Hitchcock, un Echassier dont le talon serait emplumé. Il serait à désirer que ces traces fussent étudiées par les naturalistes européens.

Quelques os signalés, mais en petit nombre, dans le terrain jurassique, à Hastings et dans le sable vert de NewJersey, également en Amérique, ont été regardés comme d'oiseaux. M. Meyer croit qu'ils sont plutôt de Ptérodactyles.

\section{$\$$ II.}

Dans les terrains jurassiques.

Sœmmering a envoyé en 1827 au Muséum le modèle en plâtre d'une plaque calcaire de Solenhofen, en Bavière, et sur laquelle est écrit tête de Héron. On y voit en effet la tête et à côté la mandibule et l'os hyoïde d'un animal qui semble être un oiseau voisin des Ardea et dont la taille serait celle du Butor. Mais l'original de ce modèle est figuré et décrit par M. Golfuss sous le nom d'Ornithocephalus (Pterodactylus) Munsteri. (1)

C'est encore à des Ptérodactyles, c'est à dire à des reptiles voisins des oiseaux, sous plusieurs rapports, qu'ont été rapportés les prétendus débris d'oiseaux nageurs que Blumenbach citait dans les schistes de Pappenheim. (2)

(1) Nova acta nat. curios., XV, p. 112, pl. YI f. 1.

(2) G. Cuvier, Oss. fossiles, III, 305; 182\%. 
Plusieurs autres indications sur les ornitholithes des terrains jurassiques n'ont pas moins besoin d'être vérifiées sur la nature même, et nous ne nous y arrêteruns pas.

\section{SIII.}

Dans les terrains crétacés.

On en a constaté avéc plus de certitude dans les formations crétacées, principalement en Angleierre. M. Mantell a reconnu pour prov nir d'un Echassier voi:in du Héron (Ardea vulgaris) un tarse, un humérus et un radius de la formation wealdienne de Tilgate-Forest (1); mais il n'en a donné ni la description ni la figure.

M. Escher, de Zurich, s'est procuré, dans les schistes du canton de Glaris, que l'on rapporte à la formation néocomienne, le squelette d'un oiseau dont on voyait distinctement l'aile et le pied. M. de Meyer (2), qui a étudié la pièce elle-même, y voit un Passereau de la taill' de l'Alouette (Alauda vulgaris).

Lord Enniskill n a découvert, près de Maidstone, en Angleterre, quelqu's os, et parmi cux un humérus qui indiquent aussi un oiseau, comme lui-mème et M. Buckland l'ont reconnu. M. Owen a parlé de cet os à la Société géologique de Londres(3), et il est autorisé à conclure, de l'étude qu'il a faite de l'humérus, que c'est un os de Palmipède longipenne de la taille d'un Albatrosse (Diomedea). Un second fragment est de tibia et fournit les mêmes iñdications.

(1) Proceed. geol. soc. London, 1833, p. 205.

(2) Bronn el Léonard, Neues iarbuch.

(j) Lrocced., 1840, p. 293. 


\section{CHAPITRE II.}

ORNITHOLITHES DES FORMATIONS TERTIAIRES MOYENNES ET SUPÉRIEURES D'EUROPE.

Le nombre en est considérable, surtout pour les Osteornis, et cependant on n'en a pas encore de tous les étages tertiaires. Quelques gisements riches en mammifères terrestres n'en ont fourni aucune trace, et dans les dépôts marins ils sont d'une grande rareté. L'étage inférieur des calcaires grossiers et des argiles en manque compléternent, du moins dans l'état actuel de nos connaissances; on ne peut cn effet supposer que les oiseaux aient manqué à l'épojue de leur formation. l.es gypses des environs de Paris et les dépôts tertiaires de l'Auvergne en ont au contraire fourni de très nombreux et de très caractéristiques, aussi commençonsnous par les ornitholithes de ces deux localités.

C'est dans des formations tertiaires seulement qu'on a recueilli des œufs et des plumes fossiles d'oiseaux.

\section{S I.}

Ornitholithes des gypses de Paris et des autres formations aux environs de cette ville.

Ils sont tous à l'état osseux et par conséquent de la catégorie des Osteornis. 
Lamanon, qui fut une des victimes de l'expédition de Lapeyrouse, a fait connaître le premier, en 1782 (1), des os d'oiseaux enfouis dans les gypses de Montmartre et sans indiquer de quelle espèce ils provenaient; $m$ ais il a parfaitement reconnu leur nature ornithologique. Un second exemplaire fut signalé en 786 par Pierre Camper (2); il consistaít en un pied.

Peu de temps après, G. Cuvier en communiqua un autre à l'Institut : il provenait de Clignancourt (3). De La Metherie a publié, dans le même recueil, une notice sur un quatrième ornitholithe de Montmartre, et deux autres personnes, MM. Goret et Burtin, redigèrent, au sujet du même morceau, leur Notice sur un oiseau fossile incrusté dans le gypse et trouvé à Montmartré, notice dont le premier présenta à l'administration du Muséum, dans sa séance du $\mathbf{1}^{\text {er }}$ prairial de la même annéeune copie manuscrite quel'on conserve à la bibliothèque de cet établissement. Deux autrespersonnes, MM. Baillet et Traullé, et plustard G. Cuvier, constatèrent aussi sur la pièce elle-même, et sur une seconde qui était également entre les mains d'un graveur d'Abbeville, nommé Elluin, que c'étaient bien des ornitholithes.

Il y avait donc, dès 1800 , ainsi que le dit Cuvier, cinq morceaux bien différents indiquant la présence d'oiseaux fossiles dans nos platrières :

Celui de Lamanon, qui appartenait à Darcet;

Celui de Pierre Camper, dont le fils de ce célèbre naturaliste a depuis lors envoyé un dessin à la Société philomatique de Paris;

(1) Journal de physique. T. 19, p. 175.

(2) Transactions philosophiques pour 1786 et Bull.Soc. philom, an V1II, p. 141.

(3) Journ de Physique, T. 51, p. 128. avec une note de Delamẻtherie, p. 152. 
Deux appartenant au graveur Elluin;

Et celui de G. Cuvier, trouvé à Clignancourt;

Tous cinq de la commune de Montmartre.

Depuis lors on en a rencontré beaucoup d'autres, et G. Cuvier en a fait le sujet d'un chapitre spécial de ses Ossements fossiles.

Dans l'édition de 1825 il en traite avec quelques détails, et comme les pieds sont plus fréquents qu'aucune autre partie; qu'ils indiquent dans plusieurs cas l'ordre auquel l'animal dont ils proviennent appartenaient, et que leurs proportions peuvent toujours conduire à quelques résultats pour la numération des espèces de ces Osteornis, c'est essentiellement d'après les pieds qu'il s'est guidé dans ce travail.

Sans prétendre arriver par les pieds seuls à la diagnose méthodique des espèces, il en reconnaît neuf, indiquées par eux, et qu'il range comme il suit, d'après leur grandeur:

I. Celle indiquée par la phalange de la planche 75 , $/ \mathrm{lg} .3$.

II. Un oiseau de proie, dont le medius, figuré pl. $74, f i g .2$, se rapproche de celui du Buzard.

III. L'oiseau auquel appartiennent l'extrémité, pl. 75 , fig. 1, et le tibia et les phalanges, pl. 73 , fig. 7 .

IV. Celui dont proviennent les pieds des fig. 4 et 6 de la planche 72.

V. Celui des pieds des fig. 9 et 11 de la même pl. 72 .

VI. Celui du pied à tarse arqué, pl. 72, fig. 5.

VII. Celui des pieds des fig. 1, 2 et 8, pl. 72 , qui est le plus abondant.

VIII. Celui de la fig. 10, pl. 72 , très voisin du précédent et paraissant appartenir comme lui à l'ordre des Echassiers. 
IX. Enfin le petit de la fig. 2, pl. 75 , qui est la plus petite des espèces recueillies jusqu'en 1825 .

Cuvier ne tire d'autre conséquence, de la distinction de ces neuf sortes de pieds, que celle de l'existence de neuf espèces d'oiseaux; mais il ne cherche pas à déterminer d'une manière plus certaine leur nature spécifique, et en effet dans la majorité des cas dont il s'agit il était impossible d'arriver à plus de précision sans risquer de compromettre le principe lui -même des déterminations palëontologiques.

On avait trouvé dès cette époque, et l'on a recueillis depuis lors d'autres os, quelques têtes ou fragments de têtes et de mandibules, ainsi que plusieurs squeiettes plus ou moins complets, et dont on pest obtenir de meilleurs renseignements. Aussi Cuvier a-t-il cherché la concordance de ceux qu'il a possédés avec ses diverses sortes de pattes.

A l'espèce IV appartiendrait un humérus (pl. $7 \overline{3}, f i g .9$ ) long de o.o'ig. Cuvier dit en effet: "Rien n'empêcherait - qu'il n'eút appartenu aux pieds de la quatrième sorte. - D'apiès sa forme il est de l'ordre des Oiseaux de rivage, " et paraît tenir de très près à celui de la Bécasse. "

A l'espèce $\mathrm{V}$ appartiendraient le squelette de la pl. 74 , fig. 1, dont il parle à la page 319 ; le bec isolé de la pl. 74 , fig. 8, et les humérus, pl. 74, fig. 9 et 10 . Cette espèce approcherait de la Caille.

A l'espèce VIII dont le pied lui avait paru, dès 1800 , se rapprocher de celui des Alouettes de mer, il réunit l'aile de la fl. $73, f i g .5$.

A l'espèce IX appartiennent à peu près certainement les deux squelettes de la pl. 75 , fig. 5 et 6 .

Quelques autres débris fournissent aussi à notre célèbre auteur des indications nouvelles pour la distinction d'un plus grand nombre d'espèces. 
X. L'oiseau de Lamanon, c'est à dire celui qui avait appartenu à Darcet, peut être, quoique les pieds n'aient pas été conservés, regardé comme d'une dixième espèce, car ses ailes et son bec étaient plus courts que dans les deux squelettes dont il vient d'être question, et les pieds de ces squelettes étant les plus petits de tous, il est bien clair, dit $\mathrm{Cu}$ vier, que cet oiseau était encore d'une espèce différente de toutes les autres. Il croit qu'un petit humérus ( $\mathrm{pl} .74$, fig. 1) lui appartient aussi.

XI. Une autre espèce est indiquée par un métacarpe (pl. 75 , fig. 5) semblable à celui du Balbuzard, et plus grand que celui du Buzard, auquel on peut au contraire comparer le médius type du $n^{\circ} I I$.

Cuvier ne donne pas de numéros aux espèces suivantes, qu'il indique néanmoins :

Deux pílicans, l'un d'après une omoplate (pl. $73, f i g .12)$, l'autre d'après un fémur ( $\mathrm{pl} .7 \overline{3}, f i g .13)$ qui vient d'use espèce bien plus grande que celle qui a fourni l'omoplate, plus grande même que le Cormoran (Pelecanus carbo), mais inférieure au Pélican proprement dit ( $P$. onocrotalus).

Un strix, d'après un métacarpe ( $\mathrm{pl} .75, f i g .4)$ à peu près de mème longueur que celui de la Chouette; d'après l'humérus de la pl. $75, / g .7$, et d'après un humérus incomplet (pl. 75 , /ig. 7 ).

Une ibis, d'après un fémur (pl. $7 \overline{3}, f i g .14$ ).

D'autres os sont encore signalés et figurés par Cuvier, mais dans le seul but de confirmer la présence d'oiseaux fossiles dans le gypse.

L.e nombre des espèces admises dans les gyps's en 1825 peut donc être porte à quinze; mais il n'y en a que neuf 
dont on ait indiqué le genre d'une manière définitive, savoir :

3 Oiseaux de proie des genres Halicatus, Buteo et Strix;

1 Gallinacé du sous-genre Coturnix;

5 Echassiers des genres Ibis, Scolopax et Pelidna;

2 Palmipèdes du genre Pelecanus.

Depuis cette époque les collections du Muséum se sont enrichies de diverses pièces importantes; celle de la collection de M. de Bournon leur ont été réunies, et quelques particuliers s'en sont aussi procuré de nouvelles (1).

Nous n'avons rien à ajouter au peu de choses que Cuvier a dites sur les Oiseaux de proie, si ce n'est que le Strix est jusqu'à présent la plus douteuse des trois espèces dont il admet la démonstration, et que les variations de la longueur du tarse dans les genres diurnes, comme dans presque toute la classe des oiseaux, permettent difficilement d'établir la proportion respective des autres parties du corps.

G. Cuvier termine ainsi son mémoire :

"Au moment où l'on achève l'impression de cette feuille, je reçois encore de Montmartre un ornitholithe où la tête, le cou, l'aile, la cuisse, et, ce qui est plus extraordinaire, la trachée artère, sont en place et bien conservés; on y distingue jusqu'aux osselets qui renforcent la sclérotique, et jusqu'à l'empreinte du cerveau. "

Des pièces nourelles qu'on voit dans les galeries du Muséum permettent d'étendre sous quelques rapports les résultats obtenus par Cuvier. Elles indiquent des Passereaux de plusicurs espèces, un Numenius voisin du Courlis (probablement l'Ibis de Cuvier), un Pelidna, un Tringa, etc.

(1) M. Duval possède un pied qui paraît être de la septième sorte. On conserve dans les collections du Collége de France un Ornitholithe dont je dois la communicalien à M. Elie de Beaumont; c'est un de ceux dont Cuvier a parlé. De lamélherie en avait cité d'autres en 1802, dans le Journal de Physique T. 55, p. 59. 


\section{Appendice aU $\$ I.}

Ornitholithes de Paris trouvés au dessus des gypses.

Nous avons dit que les gypses étaient presque le seul gisement d'Osteornis des environs de Paris, et en effet nous n'en connaissons encore ni au dessous ni dans les divers étages tertiaires qui leur sont supérieurs.

M. Duval, qui a recherché avec un grand soin les fossiles du diluvium dans une sorte de conglomerat caillouteux que l'on exploite auprès de la barrière d'Italie ou de Fontainebleau, et qui a trouvé dans ce dépôt des ossements de Blaireau, d'Eléphant, de Rhinocéros, d'Hippopotame, de Marmotte, etc., y signale aussi la présence d'oiseaux, d'après un cubitus qu'il m'a permis d'étudier.

Ce cubitus est entier, long de 0,075 . On y voit très bien les points d'insertion des pennes cubitales; c'est celui du côté gauche, et il provient d'une espèce du genre Phasianus, tel que l'établissait Linné; peut-être même est-il du genre Phasianus des ornithologistes actuels.

Un autre os également découvert par M. Duval, est une extrémité inférieure d'humérus droit. Il est moins caractéristique et ne vient pas du même sujct que le précédent. II paraît cependant du même genre, et indique une espèce ou au moins un individu un peu plus petit.

MM. C. Prevost et Desnoyers, dans leur notice sur le curieux gisement de Montmorency, où ils ont recueilli des os de Spermophile, de Cricetus et de Lagomys, indiquent aussi des débris d'oiseaux, et ils les rapprochent du Ralle d'eau commun. 
M. Duval nous en a remis un qui vient du Port-à-l'Anglais, sur la rive droite de la Seine, dans une petite formation d'apparence ligniteuse, mais qui renferme, avec des produits de l'industrie humaine, à l'époque druidique, des os de castor, sanglier, cheval, etc. C'est un fragment supérieur d'humérus indiquant une espèce de Tringa ou Bécasseau de taille moyenne.

\section{§ II.}

Ornitholithes des terrains d'Auvergne.

Une des premières indications de la présence d'ossements d'oiseaux dans les formations tertiaires de l'Auvergne est due à Cuvier, qui rapporte, en 1825 , avoir reçu de ces ossements par l'entremise du comte de Chabrol Volvic (1).

Faujas de Saint-Find avait possédé un tarse de grand Echassier, trouvé en 1812 par le docteur Boirot, dans la marne du Mont.Libre, auprès de Ganat. Ce morceau fossile est actuellement au Muséum.

Depuis une quinzaine d'années, les recherches actives de MM. de Laizer, Croizet, Bravard, et de quelques autres paléontologistes du même pays, leur ont procuré un très grand nombre de débris de la classe des oiseaux, et le $\mathrm{Mu}-$ séum en possède actuellement une suite considérable provenant de l'abbé Croizet; d'autres font partie d'un ènvoi que M. E. Geoffroy avait reçu de Chaptuzat, d'après les ordres de madame la princesse Adélaïde, et quelques autres

(1) C'est un humèrus entier des calcaires sableux et pétris de Cypris de Chapluzat. L'èliquelle morte qn ii a élé recueilli par \$. Legay. 
ont été acquis en différentes occasions. M. le professeur Jourdan en a aussi recueilli pour le musée de Lyon, et il a bien voulu nous les communiquer.

Voici à quels genres les auteurs ont rapporté ceux qu'ils ont publiés :

$a$. Un Catharthes, d'après M. Jourdan. J'en trouve la citation dans le Lethoea de M. Bronn et dans la Paléontologie de M. Pictet; mais je n'en connais pas le type, non plus que la description.

$b$. Des débris, et parmi eux des becs longrs et à pointes aiguës, analogues à ceux des Chevaliers. Ils sont indiqués par M. Demey (1), qui les a trouvés au nord de Gergovie. Le même auteur signale aussi des Gralles et des Palmipédes au sud du même plateau, au dessus du village de Merdogne, et à la page 217 du même volume, $\mathbf{M}$. Croizet reproduit son assertion, mais sans y ajouter de nombreux détails.

En 1828 , M. de Laizer (2) avait indiqué plusieurs localités à osteornis, et il avait aussi fait connaître la découverte intéressante d'œufs observés en plusieurs endroits. " Ces œufs, dit-il, sont en général brisés, mais il en est qui sont entièrement conservés, et dont la coquille est sans fracture ni fente, tt qui cependant sont remplis de la même matière qui les enveloppe. o L'auteur de cette notice en a rencontré en 1824 près du village d'Autza, dans le banc qui règne de la Sauvetas à Neschers, et aussi à côté de Perrier. M. Croizet en a possèdé plusieurs, actuellement à la collection, qui viennent de Cournon, d'Aurillac, de Gergovie et de Pont-de-Château. M. Jourdan m'en a communiqué un qu'il a recueilli à Ronzon. Ces œufs, qui sont bien cer-

(1) Bull. soc. géol., $1855-56$, p. 105 et 106.

(2) Ann. sc. nat. T.XY, p, 419. 
tainement d'oiseaux, paraissent être ceux de quelque espèce palustre; peut-être sont-ils du Flammant, dont nous parlerons plus loin.

Nous ajouterons à ce qui précède les indications suivantes :

La collection doit à M. Croizet un tarse d'Oiseau de proie, une tête de Flammant, des os d'Échassiers cultrirostres, vo sins des Hérons, deux fragments de têtes indéterminées, etc. M. Jourdan m'a prêté une partie postérieure d'oiseau que je crois de Harle, et M. Bravard, deux os, l'un recueilli par lui à Ardé; c'est une portion de tarse de Gallinacé avec son éperon; l'autre, déterré à Coude, et qui est un tarse de quelque espèce de Perdrix. Je dois dire aussi que M. Croizet a enroyé au Muséum, avec sa collection, deux portions de plumes bien reconnaissables, et que M. Poortmann, préparateur au Muséum, a recueilli avec M. Jourdan une autre plume fossile auprès de Neschers.

Le dessus de sacrum altéré, avec les os des iles, et des restes de vertèbres lombaires et dorsales provenant de Ronzon, indiquent un oiseau plongeur, et que l'allongement des os des iles nous fait regarder comme un $\|_{\text {ARLE de la taille }}$ du H. huppé (Mergus serrator). Comme nous l'avons déjà dit, il a été découvert par M. Jourdan, et appartient au Musée de Lyon.

Le fragment de tarse d'Arde qui fait partie de la collection de M. Bravard est du côté droit, et n'est que la partie la plus voisine de l'éperon, qui est long de 0,021 (quoique son sommet ait été cassé) et haut de 0,013 à sa base. Cet éperon est assez comprimé, creusé d'une gouttière près de sa base, à la face postérieure, pour le passage des tendons. A cet endroit, la face externe du tarse a 0,015 , et elle diminue brusquement à 5 millimètres au dessous de la base de 
l'éperon, par la cessation de la crête postérieure de l'os, qui n'est que la soudure au canon du métatarsien du pouce.

Ce fragment intéressant a plus de rapport avec celui du coq qu'avec la même partie chez le paon et autres Gallinacés auxquels je l'ai comparé. Il indique un oiseau de taille intermédiaire à celle du paon et du coq, mais à jambe plus comprimée encore que chez ce dernier. Je ne le crois pas de la même espèce. Le gisement d'Arde est moins ancien que ceux dont il vient d'être question; peut-être est-il diluvien. On y a trouvé quelques ossements d'hyènes, etc.

Les formations les plus récentes de l'Auvergne ont aussi des Osteornis. M. Croizet en possédait quelques-uns des Travertins, fissures ou petites cavernes de Coude.

Le tarse de Cıude que nous a communiqué M. Bravard est entier et long de 0,037 , large de 0,007 aux poulies digitales, et de $0,00 \mathrm{z}$ à l'articulation tibiale. Il ressemble à celui de nos perdrix, francolins ou petits tetras, mais il ne serait pas prudent d'affirmer qu'il provienne de telle espèce plutôt que de telle autre, parmi celles dont la taille est analogue.

\section{§ III.}

Ornitholithes des autres parties de la France.

Les plus anciens appartiennent à l'époque tertiaire moyenne et ont été recueillis par M. Lartet dans le département du Gers. M. de Blainville, dans son rapport fait en 
1837 (1), en cite de deux espèce, mais d'un genre encore à déterminer.

D'autres ont été découverts par M. de Christol et quelques autres naturalistes dans les terrains marins supérieurs du midi de la France, ce sont :

a. Des os d'Échassiers, les uns de grande taille, d'autres de celle des plus petites espèces de Hérons;

b. Des Palmipèdes, dont certains au moins sont de la taille du Cygne commun.

On connaît aussi des ornitholithes dans les gypses d'Aix, mais point encore d'osteornis; la seule indication que nous ayons est celle d'une plume que M. Coquand y a rencontré avec des poissons, des insectes, etc. (2)

Tous les autres gisements sont d'époque diluvienne, soit dépôts, soit brèches, soit cavernes. On en trouvera le détail dans l'ouvrage de M. Marcel de Serres (3). Nous rappellerons seulement qu'on n'y a reconnu, dans le petit nombre de ceux qu'on a déterminés, que des espèces actuelles, mais encore en petit nombre. Leur détermination est due à MM. Marcel de Serres, Dubreuil, Jeanjean et Puel. M. Puel désigne positivement à Brengues les Corvus pica et Perdix Cinera; M. Marcel de Serres nomme, mais dubitativement, les Anas olor et Anas anser. M. Billaudel cite le Perdix coturnix à Avison, près Bordeaux.

Le dépôt diltivien de Soute (Charente-Inférieure), dont j'ai reçu quelques ossements de loup, d'éléphant, de rhinocéros, de cheval et de bœuf, a aussi fourni quelques fragments d'oiseaux; d'autres ont été signalés dans des localités voisines.

(1) Comptes rendus Acad. $s c$.

(2) Bull. Soc. géol. 1859, p. 78.

(5) Essai sur les caternes à cssements. 
Après avoir traité des ornitholithes tertiaires et quatenaires de la France, nous devons parler de ceux que les naturaiistes ont découvert dans les autres parties de l'Europe; et c'est ce que nous allons faire, en commençant par ceux de l'Angleterre.

\section{\$IV.}

Ornitholithes de l'Angleterre.

La rareté des couches tertiaires dans ce pays ne nous permettait pas d'espérer un grand nombre d'ornitholithes. On en a cependant indiqué dans plusieurs endroits, à l'île de Sheppy, et à Norwich principalement.

M. Kœnig a signalé un crâne de Palmipède dans le London-clay de Sheppy, et quoiqu'il n'ait pas établi les caractères différentiels, il en a fait le type d'un nouveau genre, sous le nom de Bucklandium diluvir ( 1 ).

M. Owen a parlé avec détails d'autres ornitholithes du même endroit, qui font partie de la riche collection des fossiles de Sheppy, réunis par M. Bowerbank, de Londres, et comme un sternum presque entier est au nombre de ces os, l'auteur a pu déterminer le groupe réel auquel ils appartiennent. Il y reconnaît une espèce de Vautour de la taille du Cathartes aura, mais il n'en établit pas les différences par rapport au Percnoptère d'Europe. Il admet que

(1) Icones foss., n. 91; cilé par M. Pictet, T. I, de sa Paléontologie et antẻrieurement par M. Bronn. 
ces ossements indiquent un genre nouveau de Vulturidés, qu'il appelle Lithornis; Litr. vorturinus est le nom de l'espèce type de ce genre. M. Owen rapporte au Lithornis un sacrum également de Sheppy, qui faisait partie de la collection de John Hunter, actuellement le musée du collége des chirurgiens, et il en donne la figure.

Le Crag de Norwich a fourni dans ces derniers temps quelques débris non déterminés d'oiseaux réunis à des ossements de mastodonte, d'éléphant et de cheval (1).

Les cavernes de Kent, de Kirkdale et d'Oreston, ont offert des gisements plus riches d'oiseaux fossiles. M. Buckland rapporte les os de Kirkdale aux genres Corvus, Arauda, Perdix, Columba, Scolopax et Anas.

\section{$\$ \mathrm{~V}$.}

Ornitholithes de la Belgique.

Nous ne connaissons que ceux dont a parlé Schmerling, et qui sont des cavernes des environs de Liége. Voici comment il indique leur espèce :

" $1^{\circ}$ Les débris d'un Oiseau de proie (phalange ongueale grande comme celle d'un tigle).

- $2^{\circ}$ Ceux de deux espèces de Passereaux d'une petite taille, dont il est impossible de reconnaître le genre. Une portion de mandibule a des rapports avec celle du Martinet.

(1) Proceed. geol. soc. London. 
“ $3^{\circ}$ Des débris de Corbeau qui sont plus reconnaissables.

a $4^{\circ}$ Deux variétés de Gallinacés qui ont laissé leurs dépouilles dans le limon ossifère des cavernes; l'une ressemble au coq domestique; l'autre est de la grandeur d'un pigeon ordinaire.

a Et $5^{\circ}$ parmi les Palmipèdes, nous comptons deux espèces dans les débris fossiles; l'une se rapproche d'un Canard de petite taille; l'autre était de la grandeur de l'Oie orordinaire. (1)

\section{\$ VI.}

\section{Ornitholithes de la Suisse.}

Blumenbach (2) assurait déjà, dans son Manuel d'histoire naturelle, qu'on trouve des débris d'Oiseaux de rivages dans les schistes d'OEningen. Karg en a fait connaitre, en 1805 (3) qu'il regarde comme de Bécasse (Scolopax) et depuis lors MM. Aman et Schinz, cités par M. Bronn, ont rapporté le même fait.

Les mollasses de la Suisse, qu'on a étudiées avec soin dans ces dernières années, ont fourni quelques Ornitholithes.

M. Bourdet, cité par M. Bronn, a obtenu au mont de la Molière des os qu'il regarde comme provenant d'un Coq (GaLlus).

(1) Oiseaux foss. de la province de Liüge, 13. 172. Les osscments sont figurés à la planche 27 .

(2) Manuel, T. II, p. 408, de l'éd. franc.

(3) Ecrits des naluralistes de Souabe, 1, pl. 2, fig. 1. 


\section{$\$$ VII.}

\section{Ornitholithes de l'Allemagne.}

Dans son rapport à l'Association britannique pour l'avancement des sciences (année 1843), M. Murchison parle de treize espéces d'oiseaux du bassin tertiaire du Rhin moyen observées par MM. Meyer, Kaup et Bronn, mais il ne dit rien de plus à cet égard (1).

Antérieurement, M. Hœninghaus avait signalé un fémur de Cormoran dans le calcaire supérieur d'eau douce, auprès de Creteld, sur le Rhin (2). Depuis lors, il a parlé d'une côte; également d'Oiseau de marais, suivant lui, et trouvée au même lieu. On possède aussi quelques notes sur des oiseaux de l'époque diluvienne recueillis en Allemagrne.

M. Bronn, dans son Lethcea, cite d'après M. Germar, un Vultur de Westeregeln, trouvé avec du Rhinocéros minutus;

Et d'après Schlotheim, un Fulica des lignites (Braunko$h(e)$, à Kaltennordheim.

On a encore signalé des ossements d'oiseaux à Canstad, etc.

\section{$\mathbb{S}$ VIII.}

Ornitholithes du midi de l'Europe.

Les terrains du val d'Arno paraissent avoir donné aux

(1) L'Instilut.

(2) Bull. Soc.géol. de France, 1839, p. 26. 
observateurs italiens quelques débris fossiles d'oiseaux, mais qu'on n'a pas déterminés. L'analogie de ces terrains avec les couches tertiaires supérieures de l'Auvergne, doit faire désirer que ce travail soit entrepris par quelque naturaliste.

Les brèches méditerranéennes d'Italie renferment, comme celles de Cette (1) et de Corse, des Osteornis. On cite un oiseau semblable aux Turdus, et un autre aux Larus, dans les brèches de Nice, et M. Wagner (2) a étudié ceux de la Sardaigne. Il y reconnaît :

Falco (Buteo) pennatus; Strix nyctea; Corvus de la taille du $C$. corone; Corvus corax; Turdus merula?; Aladda! Fringilla; Picus martius?; Anas boschas.

L'Italie est du petit nombre des pays qui ont fourni des plumes fossiles. Faujas de Saint-Fond a depuis longtemps décrit (3) deux plumes fossiles de Vestena-Nova (MonteBolca), dans le Véronais, que l'on conserve encore au Muséum. Elles viennent des mêmes couches que les nombreux poissons figurés par Volta dans son Ichthyologie véronaise.

(4) «Les oiseaux, dit Cuvier en parlant des brèches de Cette, t. Iv, p. 179, m'ont èté annoncès par une seule moitié inférieure du cubitus, mais que personne ne peut méconnaître, quand ce ne serait qu'aux petites élévations qui servaient d'attache aux plumes. Son articulation inférieure et sa grandeur correspondent à celles de la Bergeronnette et d'autres Passeres. "

(2) Mém. de l'Acad. de Munich pour 1832.

(3) Ann. du Druseum, IIl, p. 18, pl. 1, fig. 1-3. 


\section{CHAPITRE III.}

\section{ORNITHOLITHES ÉTRANGERS A L'EUROPE.}

Nous aurons à parler successivement, dans ce chapitre, des ossements qu'on a recueillis dans le nord de l'Asie, dans l'île Rodrigue, située près Bourbon, en Amérique et à la Nouvelle-Zélande. Tous sont de l'époque tertiaire supérieure ou même alluviale, et plusieurs d'entre eux sont très remarquables, autant par leur grande taille que par la singularité de leurs caractères anatomiques; aussi leurs espèces ont-elles été établies avec une véritable certitude.

\section{SI.}

Gryphus antiquitatis.

Le seul ouvrage français qui parle encore de cet oiseau est dû à M. Ami Boué (1); il nous apprend seulement qu'il a été découvert dans les glaces de l'île de Lachow, et sur les côtes asiatique et américaine, près du détroit de Behring. Nous voyons par la liste des oiseaux fossiles dil Lethoea de M. Bronn, que le Gryphus appartient au groupe des Vautours, et qu'il est du diluvium sibérien; mais nous

(1) Guide du géologue voyageur, II, 25\%; 1836. 
ignorons ses caractères. M. Schiff nous a dit qu'on en possédait quelques ossements au Musée de Munich.

\section{\ II.}

Du Dronte et du Solitaire des îles Mascareignes.

G. Cuvier a présenté à l'Académie des sciences, en 1830 , des os incrustés de Stalactites que lui avait envoyés feu M. Desjardins, de l'île de France, et qu'on avait trouvés dans l'île Rodrigue, située dans la mer des Indes, à l'est des Mascareignes. " Il a supposé qu'ils pouvaient provenir du Dronte, et, dans tous les cas, d'après leur forme et surtout d'après celle du crâne, du sternum, du très petit humérus, du fémur et du tarse, il a juggé qu'ils appartenaient à un oiseau apparenté aux Gallinacés. „ (1)

M. de Blainville, qui a rédigé son mémoire sur le Dronte à l'époque où Guvier fit cette communication, dit en terminant son travail sur cet oiseau célèbre :

- Il y aurait maintenant une autre question à traiter, savoir si les ossements incrustés qui ont été envoyés dernièrement de lî̉le de France à $M$. Cuvier, ont réellement appartenu au Dronte, comme M. Cuvier a été porté à le croire. C'est une question qu'il sera de la plus grande facilité de résoudre par la comparaison immédiate de ces os arce les pièces conservées en Angleterre. Si cela était, ce que la différence de hauteur de l'os du tarse ne permet pas de

(1) Hist. des progrès des sc. nat., T. V̀, p. $407 ; 1836$. 
croire, il serait en même temps prouvé que le Dronte existait aussi à Rodrigue, car ces ossements ont été trouvés dans cette île, dans une grotte, comme me l'a assuré M. Quoy, qui les avait vus à son passage à l'île de France, et non pas à l'île de France, sous des couches de laves, comme l'a dit M. Cuvier, sur des renseignements erronés dans sa note lue dernièrement à l'Académie. Alors il y aurait une presque certitude. "

Ces os sont au nombre de six :

Une boite crânienne sans bec ni mandibule;

Une portion considérable de sternum;

Un humérus droit entier;

Un fémur gauche entier;

Un tarse entier;

Un cubitus entier.

Quand au Dronte lui-même, le Didus ineptus de Linné, comme les pièces tout à fait précieuses sur lesquelles repose sa connaissance actuelle n'ont réellement pas d'intérêt géologique, puisqu'elles ont été préparées d'après le frais, nous n'avons pas à en parler ici. On trouvera d'ailleurs dans Buffon et dans le mémoire bien connu de M. de Blainville, tous les renseignements désirables à l'égard de cet oiseau. Il est néanmoins de notre sujet de rappeler que la race des Drontes de l'île de France, comme celle des Solitaires de Rodrigue, est actuellement éteinte, et qu'un temps assez court a suffi à leur destruction. On doit donc y voir, avec $M_{\mathrm{e}}$ de Blainville, " la preuve manifeste qu'un animal peut disparaître du nombre des êtres actuellement vivants, et cela, de nos jours, presque sous nos yeux, et sans qu'il y ait besoin d'autre catastrophe que l'avidité irréfléchie de 
l'espèce humaine, et son extension dans une partie du monde où cet animal semblait être limité. n

Le Dronte et l'Oiseau de Rodrigue étaient sans doute de la même espèce, et certainement cette espèce n'appartenait pas à l'ordre des Cursores. Ce n'était pas non plus un Gallinacé comme l'a dit Cuvier, mais un oiseau lié en même temps aux Vautours et aux Gallinogralles les plus voisins de ceux-ci, par exemple les Kamichis.

\section{S III.}

Ornitholithes de l'Amérique meridionale.

M. Lund en signale dans les mêmes terrains qui lui ont fourni tant de curieux mammifères, et parmi eux il cite des os d'une Autruche du genre Rires, plus grande que les deux espèces actuelles.

Cet Observateur porte à trente-trois le nombre des espèces fossiles d'Oiseaux qu'il a recueillis dans les terrains mégathériens du Brésil (1). Ces Oiseaux sont de 26 genres, et se répartissent ainsi dans les différents ordres :

1 Oiseau de proie;

18 Passereaux;

6 Grimpeurs;

4 Gallinacés;

5 Riverains.

Ils appartiennent presque tous, comme les Mammifères fossilifiés avec eux, à des genres particuliers à l'Amérique

(1) Bronn et Leonard, Neues iarbuch, 1843, p. 237. 


\section{$-34-$}

méridionale, aux Anabates, Dendrocolaptes, Opetiorhynchus, Crypturus el Rhea.

Quelques-uns ne sauraient également être distingués comme espèces d'à présent, tels sont les Cypselus collaris, anabates polyocephalus, Capito melanotus, Coccyzus cayanus, Perdix dentata, Crex minuta.

Parmi les espèces perdues, l'une des plus remarquables par sa grande taille est de la famille des Alectoridés ou Hoccos.

M. Claussen, qui revient du Brésil où il a aussi recherché des fossiles avec un grand soin, m'apprend qu'il a un grand nombre dossements d'Oiseaux, mais il n'a point encore eu le temps de les déterminer suffisamment. Il a néanmoins reconnu ceux d'un Cathartes plus grand que les espèces actuelles; d'un Strix, d'un Caprimulgus, d'un genre voisin des Dicholophus, et, ce qui n'avait pas encore eu lieu jusqu'à lui, d'un Psitracus ou Perroquet.

\section{\$IV.}

Dinornis de la Nouvelle-Zélande.

Les intéressantes recherches que Richard Owen a faites sur les grands ossements d'oiseaux que MM. Rule et W. Williams ont recueillis à la Nouvelle-Hollande, nous ont fait connaître une série de cinq espèces de Cursores, les unes plus grandes, les autres plus petites que les Casoars, et que toutes paraissent d'une organisation intermédiaire à celle des Casoars et des Aptéryx; M. Owen leur donne le nom générique de Dinoris. 


\section{$-35-$}

Voici en quelques mots l'histoire de cette importante découverte:

M. Rule, pendant son séjour à la Nouvelle-Zélande, entendit parler aux naturels d'un oiseau qu'ils comparaient à un aigle de grande taille, mais qu'aucun individu de la génération actuelle n'a vu. La tradition rapporte que cet oiseau, auquel ils donnent le nom de movie ou moa, est une sorte de monstre vivant caché dans une caverne inaccessible d'une montagne auprès de la rivière de Wairoa, et qu'il est gardé par un lézard. Des os qu'on trouve à peu de distance presque à la surface du sol furent cités par les naturels à l'appui de leur assertion, et M. Rule s'en procura un. C'est sur l'inspection de cet os, qui était un fragment de fémur, que $M$. Owen reconnut, en 1839 , une espèce de l'ordre des Cursores, restée jusqu'alors inconnue aux naturalistes, et qui arait probablement cessé d'exister, mais à une époque assez peu reculée pour que l'espèce humaine en ait pu conserver le souvenir; le Moa n'était donc pas une espèce d'Aigle, comme le disaient les Nouveaux-Zélandais. M. Owen publia une description et une figure de cet ossement (1), et nous en avons parlé, d'après lui, dans le Supplément au Dictionnaire des sciences naturelles. (2)

Plus récemment, un magnifique envoi de ces ossements fossiles a été fait à M. Buckland par M. W. Williams, et ils ont fourni à M. Owen le sujet d'un travail étendu et accompagné de planches nombreuses (3); dans ce travail l'auteur démontre cinq espèces de ces oiseaux, et il les place toutes dans le genre nouveau qu'il a nommé Dinornis; ce sont les

D. GIGANTEUs, de 4 mètres et plus de hauteur;

(1) Proceed. zool. Soc. Lond., 1859, p. 169, et Trans. zool. Soc. Lond., p. 353.

(2) T. I, p. 525.

(3) Trans. zool. Soc. Lond., t. III, p. 23̋̈-275. pl. xviij-xxx; 1844. 
D. Ingens, de la taille de l'Autruche. M. Laurillard en a parlé d'après une note que lui avait communiquée 1 . Owen sous le nom de $D$. struthioides (1);

D. DRoмozoïds, de la taille du casoar de la NouvelleHollande;

D. DIDIFormis, à peu près aussi lourd mais un peu plus fort que le Dronte;

D. otidiformis, de la taille de notre grande Outarde.

M. le capitaine Bérard, de la marine française, s'est procuré trois os de Dinornis et les a envoyés au Muséum. Les renseignements qu'il a recueillis dans le pays prouvent qu'en certains lieux ces ossements des Moa sont très nombreux.

(1) Dict. univ. d'hist. nat., publié par M. Ch. Dorbigny, t. v, p. 33. 


\section{RÉSUMÉ.}

Les indices fossiles de l'existence des oiseaux reçoivent le nom d'Ornitholithes, quelle que soit leur nature.

Ils sont de quatre sortes principales:os, cufs, plumes et empreintes laissées par les pieds lors de la marche des oiseaux sur des terrains encore mous.

Les seules empreintes connues ont été signalées par M. Hitchcock, qui leur a donné le nom collectif d'Ornitichnites.

La signification de ces 'empreintes n'a pas encore été démontrée d'une manière suffisamment scientifique. ( 1 )

Les ossements fossiles d'oiseaux ne sauraient conduire dans tous les cas à la détermination précise de l'espèce dont ils proviennent.

On n'en reconnaît assez souvent que le genre linnéen; dans d'autres cas, ils indiquent seulement la famille, l'ordre ou même la classe.

On n'a déterminé spécifiquement qu'un très petit nombre d'Oiseaux fossiles, et ces espèces son $t$ surtout des terrains supéricurs. Ce sont les seules que l'on doive dénommer d'après les principes de la nomenclature linéenne.

Les os fossiles d'oiseaux dont on ne, peut avec certitude caractériser l'espèce, même en les comparant avec leurs analogues dans les oiseaux actuels et dont on reconnaît

(1) Des observations récentes de MM. James Deane et Lyell, citées en 1845 par M. Murchison, dans son résumé des travaux de la Société géologique de Londres, permeltent d'espérer la solution prochaine de ce problème. 
seulement le genre, la famille ou l'ordre, pourront prendre le nom collectif d'Osteornis. Un nom qualificatif ajouté à celui-ci indiquera par approximation la nature des oiseaux que,ces Ostéornis font supposer, mais sansque les zoologistes doivent cependant les introduire comme autant d'espèces constatées dans les catalogues méthodiques.

M. Adolphe Brongniart a depuis longtemps et avec avantage suivi un mode analogue de nomenclaturc dans ses savantes recherches sur les végétaux fossiles.

Les données géologiques auxquelles peut conduire l'étude des oiseaux fossiles dans l'état actuel de la science sont moins nombreuses et moins importantes que celles tirées de la connaissance des Mammifères, des Reptiles, des Amphibiens ou des Poissons.

Les terrains de transition dans lesquels on n'a encore rencontré aucune trace de Reptiles n'ont pas offert non plus d'Ornitholithes.

Dans les terrains secondaires les Ornitholithes sont jusqu'ici peu nombreux; ils y sont de deux catégories distinctes :

Ornitichnites des nouveaux grès rouges;

Osteornis des Wealds, des argiles néocomiennes et de la craie supérieure.

Quoi qu'on ait rapporté les Ornitichnites à des Échassiers, ét plus particulièrement à des Cursores, leur nature, comme nous l'avons déjà dit, n'est que très vaguement appréciée.

Les Ostéornis de l'époque secondaire peurent être dénommés ainsi qu'il suit :

O. ardeaceus, d'après M. Mantel;

O. scolopacinus, d'après M. Meyer;

O. diomedeus, d'après M. Owven.

Les Ornitholithes des terrains tertiaires sont plus nom- 
breux; on les recueille principalement dans des formations d'eau douce.

Il y en a dont l'espèce a pu être déterminée :

Numenius gypsorum, des environs de Paris, que nous regardons comme une espèce éteinte;

Phœnicopterus ruber? des terrains d'Auvergne, qu'il est impossible, d'après les os qu'on en possède, de séparer de l'espèce actuelle encore aujourd'hui vivante dans les parties méridionales de l'Europe et en Afrique.

La plupart des autres ne se distinguent pas suffisamment de leurs congénères actuels pour qu'on les retire de la caté. gorie des Osteornis, c'est à dire des Ornitholithes dont on ne peut assurer précisément l'espèce. En suivant le mode de nomenclature que nous proposons il sera facile de les dénommer d'une manière suffisante pour les besoins actuels de la paléontologie. Ce système de nomenclature nous paraît avoir un avantage non moins important, en ce qu'il ne fera point dire à la paléontologie plus que l'anatomie zoologique sur laquelle elle s'appuie, n'est réellement en droit de démontrer dans les cas dont est question.

L'ordre des Préhenseurs ou Perroquets et celui des Coureurs, les seuls qui n'aient pas de représentants actuels dans la Faune européenne, sont aussi les seuls qui manquent à la Faune tertiaire.

Les données que fournissent les autres classes de vertébrés doivent faire supposer que ces deux ordres d'oiseaux ont néanmoins existé en Europe, comme ils existent encore aujourd'hui dans les autres parties du monde : en Afrique, dans l'Inde insulaire, à la Nouvelle-Hollande, et dans l'Amérique méridionale.

Il est encore impossible d'indiquer à quelle époque la classe des oiseaux a commencé d'exister sur le globe terrestre, toute conclusion fondée étant en effet rendue impos- 
sible par l'imperfection de nos connaissances à cet égard.

On démontre cependant que les Reptiles ne sont pas, comme on le dit quelquefois les animaux vertébrés les plus élevés en organisation qui aient vécu pendant la période secondaire, puisque les oiseaux y étaient leurs contemporains.

Les oiseaux de l'époque diluvienne dont on a déterminé l'aspèce ne différaient point de ceux qui vivent encore aujourd'hui en Europe, et si l'on démontre parmi eux quelque espèce perdue, cette espècè ou ces espèces seront sans doute des oiseaux à vol lourd ou impossible, comme le Dronte qui a été détruit depuis un temps assez peu reculé à l'Isle-de-France, ou comme les Dinornis de la NouvelleZélande. 
PROPOSITIONS

DE

BOTANIQUE. 


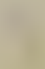

10

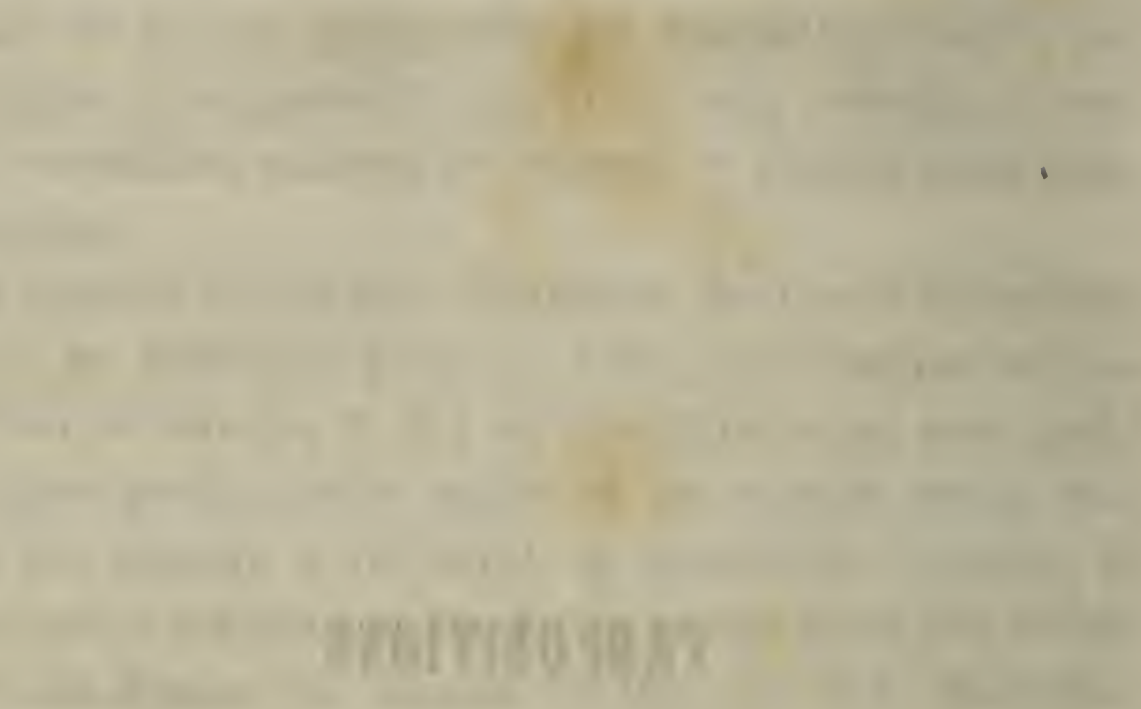

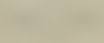

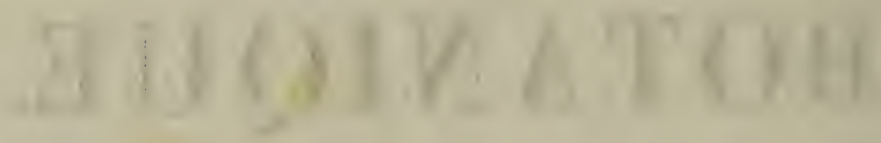




\section{PROPOSITIONS}

DE BOTANIQUE.

\section{I.}

L'état actuel de la science ne permet pas d'établir la limite précise qui sépare le règne végétal du règne animal.

II.

Il y a des végétaux qui sont doués de mouvements propres, soit dans la totalité de leur être, soit dans quelqu'une de leurs parties.

\section{III.}

Réciproquement, quelques animaux inférieurs manquent d'une cavité spéciale de leur tégument extérieur, que l'on puisse considérer comme digestive, et ils n'ont montré ni système nerveux ni système musculaire distincts.

\section{IV.}

Les spores de certaines algues et de quelques ulves qui jouissent de la propriété de translation, doivent cette propriété à des parties ciliaires de leur périphérie, découvertes par MM. Unger et Thuret, et que l'on n'a pu distinguer des cils propres aux infusoires animaux.

\section{V.}

Les Diselmis, généralement classés parmi ces derniers, ont la plus grande analogie apparente avec les spores ciliées des algues.

\section{VI.}

Les éléments textulaires des végétaux dérivent tous de la forme utriculaire. 


\section{VII.}

On peut admettre avec M. Schwann, qu'il en est de même pour ceux des animaux.

\section{VIII,}

Toute utricule est initialement de forme sphérique ou sphéroïde.

\section{IX·}

La présence d'un nucleus dans les utricules ne peut être considérée comme générale. Il manque constamment dan s celles des végétaux cellulaires; il ne se montre pas dans la formation du cambium.

\section{X.}

Les utricules peuvent être regardées comme des individualités simples, en zoologie aussi bien qu'en botanique, et cependant les végétaux ou les animaux qu'elles constituent par leur réunion 'sous une forme déterminée, sont les véritables individus botaniques et zoologiques.

\section{$\mathbf{x I}$.}

La forme sphéroïdale est non seulement la forme initiale de toute utricule, elle est encore celle de toutes les réunions d'utricules, c'est à dire de tous les animaux ou végétaux, au commencemenr de leur existence.

\section{XII.}

Cette forme est même définitive chez les végétaux comme chez les animaux les plus simples.

\section{XIII.}

Elle n'est que transitoire chez ceux dont l'organisation est plus compliquée. 


\section{XIV.}

Certains végétaux inférieurs appartenant à la famille des Diastomacés constituent par la binarité de leur forme une exception aux lois de la morphologie des végétaux inférieurs. On pourrait les considérer en grande partie, comme des réunions radiaires d'individus binaires, ceux-ci n'étant alors que des cellules modifiées dans leur forme, en vue d'un mode spécial d'aggrégation en systèmes ivdividuels.

\section{$\mathrm{XV}$.}

La forme des êtres organisés dans ses faits spéciaux et généraux semble soumise à des lois aussi précises, mais plus élevées que celles qui régissent les formes minérales.

\section{XVI.}

Les zoologistes modernes avaient réuni aux animaux un assez grand nombre de productions dont la nature végétale est actuellement démontrée. Toutes celles que M. de Blainville a séparées des animaux sous le nom de Pseudozoaires Calciphytes et Nématophytes, sont dans ce cas.

\section{VII.}

M. Decaisne en faisant connaître la nature des corps reproducteurs des Calciphytes, dont il a démontré l'identité ave la fructification des Algues, a levé tous les doutes à cet égard.

\section{XVIII.}

Les Spongiaires sont le point de contact le plus évident des deux règnes animal et végétal. 


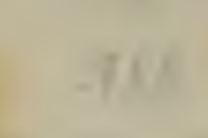

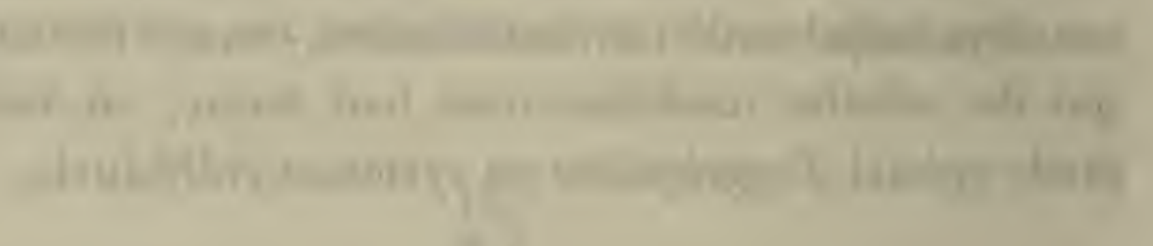

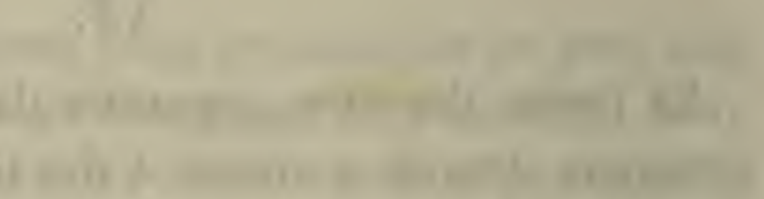

(12x

iris

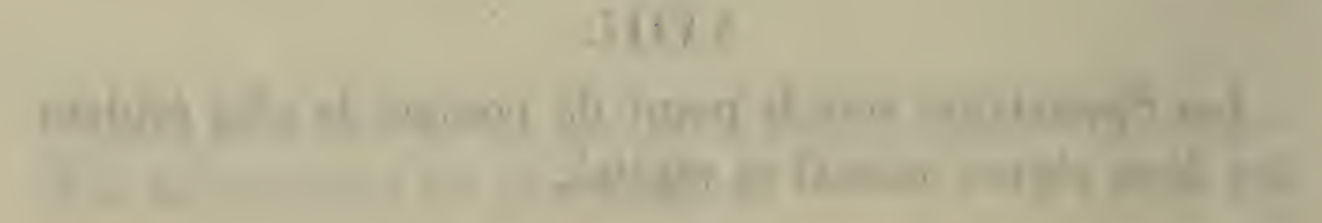




\section{$V$ u et approuvé :}

Le doyen de la Faculté des sciences,

DUMAS

\section{Permis d'imprimer :}

L'Inspecteur-général des Études, chargé de l'administration de l'Académie de Paris,

\section{ROUSSELLE.}


